ojs.uv.es/index.php/qdfed

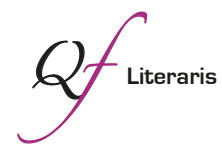

\title{
La influencia cultural china en la España moderna: encuentros $y$ desencuentros
}

\author{
The Chinese cultural influence in modern Spain: \\ points of convergence and divergence
}

\author{
Min Sun ${ }^{\mathrm{a}} \&$ José María Escribano Angulo ${ }^{\mathrm{b}}$ \\ a Universidad Complutense de Madrid.msun@ucm.es \\ b Universidad Complutense de Madrid.jmescribano20@hotmail.com \\ Recibido: 15/03/2018. Aceptado 10/09/2018
}

Resumen: La influencia que ha ejercido la cultura china en España puede ser considerada como escasa en términos generales, a pesar de los contactos directos que se produjeron entre estas dos naciones desde el siglo XVI. En este artículo se exponen los motivos de dicho encuentro y desencuentro, ya que los primeros contactos prometían una interrelación más profunda de la que se produjo. Entre estos últimos motivos de influencia destacan algunos temas literarios que quedaron como tópicos en la literatura del Siglo de Oro español y en la literatura europea posterior, y que reflejan el devenir de la influencia china en Occidente.

Palabras clave: cultura china; cultura española; Ruta de la Seda; influencia cultural.

\begin{abstract}
Chinese culture can be considered to have had little influence in Spain, despite the direct contact between these two nations from the $16^{\text {th }}$ century onward. This article outlines the grounds for the convergence and divergence, as the initial contact promised a deeper interaction than what took place. Among the latter grounds for influence, some literary themes stand out which ended up becoming clichés in the literature of the Spanish Golden Age and in subsequent European literature, and which reflect the course of Chinese influence in the West.
\end{abstract}

Keywords: Chinese culture; Spanish culture; Silk Road; cultural influence.

》) Sun, Min \& Escribano Angulo, José María. 2018. "La influencia cultural china en la España moderna: encuentros y desencuentros". Quaderns de Filologia: Estudis Literaris XXIII: 79-94. doi: 10.7203/qdfed.23.13445 



\section{Introducción}

Cuando el Imperio español se encontró con el Imperio chino, ambos estaban en su época áurea. Por aquel entonces, la Monarquía Hispánica, bajo el reinado de Felipe II (1556-1598), abarcaba extensos territorios a lo largo de todo el mundo; China, bajo el reinado del emperador Wanli (1563-1620), dinastía Ming, podría considerarse también en su apogeo territorial. Al esplendor histórico de dominio, jurisdicción y autoridad siguió, consecuentemente, un esplendor cultural que dejó su impronta, por supuesto, en la literatura.

En China, a mediados del siglo XVII, habían aparecido ya tres de sus cuatro grandes novelas clásicas, a saber, 《三国演义》 (Romance de los Tres Reinos, 1330), 《水汻传》 (A la orilla del agua, ca. 1373), 《西游记》 (Viaje al Oeste, 1590); también, tres grandes novelas cortas en Baihua -la escritura simplificada del chino tradicional Wenyan-, 《喻世明言》 (Historias para iluminar el mundo, 1620), 《警世通 言》 (Historias para apercibir al mundo, 1624), 《醒世恆言》 (Historias para despertar al mundo, 1627); al igual, circulaba la gran novela 《金瓶梅》(El ciruelo en el vaso de oro / El loto de oro, ca. 1610).

En España, por otro lado, la literatura gozaba de su máximo esplendor literario. Curiosamente, hubo referencias a China en casi todas las grandes figuras del Siglo de Oro, aunque esta influencia, no obstante, quedara limitada a ciertos tópicos sin que existiera una asentada transmisión -una relación directa- de préstamos literarios entre las dos culturas. Cuáles fueron y por qué no se produjo una influencia más profunda entre las dos literaturas es una de las cuestiones que queremos plantear en este artículo.

\section{Los primeros vínculos}

Las primeras referencias del sudeste asiático y, más allá, de China ${ }^{1}$-las Indias Orientales y Catay desde la perspectiva geográfica del siglo XVI

\footnotetext{
${ }^{1}$ Hemos optado por utilizar los siguientes términos para aludir a los referentes socioculturales de los que estamos tratando: para referirnos a China, como foco irradiador cultural dentro de Asia Oriental, a veces utilizaremos también expresiones referenciales como Extremo Oriente o Lejano Oriente; para referirnos a España, también utilizaremos el de Occidente. Somos conscientes de la dificultad de referirnos a realidades histórico-culturales diversas en tiempo y espacio, por ejemplo, resulta problemático
} 
y XVII- se remontan al periodo helenístico y al intento frustrado de la monarquía global de Alejandro Magno y su búsqueda del mar Océano. Después, bajo la PAX ROMANA del siglo II d.C., quedaron registrados ciertos contactos diplomáticos en la época de Marco Aurelio, posibilitados también por el creciente comercio de la seda, artículo de lujo para la clase acomodada romana; estas relaciones, sin embargo, fueron débiles debido a la intermediación que ejercía el Imperio Parto.

Otras alusiones posteriores provienen del mito del Preste Juan, reysacerdote de un supuesto reino cristiano de Oriente que sobresalía por sus riquezas y maravillas, en el que habría realizado su evangelio Santo Tomás. Además, en la Baja Edad Media, cobró notable importancia la literatura de viajes; hay referencias a China en el Libro de Viajes de Benjamín de Tudela y, por supuesto, en los Relatos maravillosos de Marco Polo, que constituyen, junto al citado Preste Juan, la fuente del imaginario conceptual sobre una China de carácter grandioso, hiperbólico, riquísima, tanto en la Europa medieval como en la renacentista. A todo esto hay que añadir el efecto que pudo tener una supuesta embajada comercial china a Europa perteneciente a la inmensa flota de Zheng He.

Como se puede observar, existe una especie de colusión entre aspectos que pudieron tener una realidad histórica y aquellos que son leyendas sobre el Oriente. El carácter comercial de las caravanas de objetos y especias asiáticas llegadas a Europa a través de la Ruta de la Seda produjo un interés económico relevante que alimentaba este imaginario de lugar maravilloso. El comercio, monopolizado en el Mediterráneo por genoveses y venecianos, decayó con el cierre de las rutas comerciales centroasiáticas después de la conquista de Bizancio por los turcos, hecho que produjo o estimuló el descubrimiento de América. Es curioso

que el interés principal que movía a Colón a adentrarse en lo desconocido fuera el comercio de la pimienta.

\section{El tópico chino sobre Angélica la Bella en la literatura española}

La figura de Angélica la Bella tiene su referente inmediato en las narraciones de los viajes Marco Polo. Angélica la Bella, hija del emperador

oponer el sintagma "cultura china" al de "cultura española" porque este último queda englobado en la más amplia "cultura occidental". Igualmente, se utilizarán términos como "país" o "nación" para ayudarnos a identificar los referentes. 
de Catay, es una figura que se intercala en los ciclos caballerescos europeos a través de los poemas épicos Orlando innamorato (1486) de Matteo Maria Boiardo y Orlando furioso (1532) de Ludovico Ariosto.

En España la influencia del ciclo caballeresco y la figura de Angélica es notable debido al modelo que supuso el Renacimiento italiano para nuestros escritores. El imaginario literario de nuestro periodo áureo está plagado de referencias a este personaje. En la obra teatral Las lágrimas de Angélica ([1586] 1981: 92) de Luis Barahona de Soto, se resalta su exotismo de extraña belleza:

Bellas redes de amor, madejas de oro, sartas de aljófar, púrpura, ámbar, nieve, del celebrado rostro, a quien se debe la singular belleza de Medoro, rendíos al santo y venerable coro del rojo Apolo y las hermanas nueve, quien es bien que el mundo y su riqueza apruebe lo que da el cielo por mayor tesoro.

$\mathrm{Y}$ así como linaje y fortaleza pospuso a la caduca hermosura, la antigua reina del Catay señora, posponga y rinda la mortal belleza al vivo ingenio y ciencia eterna y pura, y venza al fuerte y bello el sabio agora.

En el "Romance XXVIII" de Quevedo se recoge también esta figura:

Bella reina de Catay, heredera de la China, por quien hoy andan enhiestas tanta lana y tanta pica.

También Lope de Vega traslada el tópico al teatro en su Angélica en el Catay ([1617] Acto I, 2009: 226):

Yo soy hija del Rey, y por mi agora, digna de serlo tuya, y no he venido, con Agramante â ser encantadora, pues armas, y no echizos he traydo, es el Catay mi patria, mi ley Mora, 
y aunque lo soy, ningún mortal nacido, se alabarâ, que amor le tuue, ô tengo, oy afrentarlos por tu lengua vengo.

El carácter de la grandiosidad y de la riqueza relacionadas con China se acaba asimilando como un proceso inconsciente desde lo real-social a la manifestación literaria. Tal como comenta Manel Ollé (2000: 1):

Durante el siglo XVI [se produce] un proceso de invención de China en el doble sentido que el vocablo tenía en la época: descubrimiento y proyección sobre las realidades conocidas de primera mano de un imaginario que obedece a leyes propias, superpuesto a la percepción sin mediatizaciones.

\section{Los primeros tratados sobre China}

La expedición de Legazpi arribó a las Islas Filipinas en 1565, donde se produjo el primer contacto entre los españoles y los sangleyes -inmigrantes de origen chino-. Los motivos primordiales que estimularon la exploración de aquellas tierras fueron la búsqueda de riquezas y el desarrollo del comercio, la ambición política de poder y la transmisión de la fe católica, ideario de los monjes dominicos. El último también puede considerarse desde la perspectiva del primero: el afianzamiento del poder político.

España creyó en el común destino de la humanidad, y con ansias incontenibles de redención, se entregó a la ingente tarea de unificarla dentro del mismo espíritu y doctrina que enseña a todos los hombres la igualdad de su origen y también de sus derechos (Sanz, 1958: 20).

No obstante, aunque en China fracasara la misión evangelizadora, los misioneros ejercieron una gran influencia en la comunicación cultural entre el Occidente y el Lejano Oriente; ellos formaron la fuerza principal de divulgación de obras chinas a las lenguas europeas y buscaron coordinar la fe católica con el Confucianismo para la conquista espiritual de todo un pueblo. Si el descubrimiento de América y Asia inicia el primer paso de la globalización y el incipiente desarrollo del capitalismo, podemos considerar que los misioneros de los siglos XVI 
y XVII fueron los intelectuales que llevaron a cabo esta transmisión e hicieron de puente entre dos mundos.

La influencia, por lo tanto, fue notable y se dejó sentir en muchos ámbitos culturales. El mismo Cervantes expresaba su deseo de que Don Quijote (1616, "Dedicatoria", 2. ' parte) se leyera en el gran imperio del Lejano Oriente:

Enviando a vuestra excelencia los días pasados mis comedias, antes impresas que representadas, si bien me acuerdo, dije que don Quijote quedaba calzadas las espuelas para ir a besar las manos a vuestra excelencia; [...] y el que más ha mostrado desearle ha sido el grande Emperador de la China, pues en lengua chinesca hará un mes que me escribió una carta con un propio, pidiéndome, o, por mejor decir, suplicándome se le enviase, porque quería fundar un colegio donde se leyese la lengua castellana, y quería que el libro que se leyese fuera el de la historia de don Quijote. Juntamente con esto me decía que fuese yo a ser el rector del tal colegio.

Aunque el uso de la ironía y la hipérbole en Cervantes es generalizado, su alusión a China ha dado pie a numerosos estudios. Es probable que en su etapa de estudiante en Córdoba (1555-1556) y en Sevilla (1563-1564) conociera obras escritas por misioneros de renombre. Resulta imprescindible mencionar aquí a Francisco Javier (1506-1552). El santo navarro murió intentando llevar a cabo una embajada en China, después de su viaje de evangelización a Japón. Su vida fue celebrada y se convirtió en tema literario para el teatro jesuítico del Siglo de Oro y también para algunas comedias largas, como la Comedia de San Francisco Javier, el sol en Oriente (1691) de Diego Calleja.

Otra obra destacable fue la de Juan González de Mendoza (15451618), Historia de las cosas más notables, ritos y costumbres del gran reyno de la China (1585, Roma). En esta obra, de carácter verdaderamente enciclopédico, se recopilan multitud de informaciones y leyendas sobre el imperio asiático. Una vez publicada, tuvo tanta repercusión que provocó mucho interés hacia todo lo chino en Europa, tanto es así que sufrió una constante reedición y traducción a muchas lenguas europeas. Es muy probable que Cervantes y otros literatos de la época leyeran o tuvieran, al menos, noticia de este libro.

Las obras que circulaban en España sobre China se pueden dividir en dos tipos: libros escritos por los misioneros sobre conocimientos 
generales y, por otro lado, traducciones de libros de diversas materias, tanto literarios como de filosofía, de medicina, de agricultura, etc.

Aparte de un temprano manuscrito anónimo, Abecedario chino (s.f.), que lista los caracteres de esta lengua en alfabeto latino, señalando incluso su tonalidad, hay que destacar como uno de los pioneros al dominico Miguel de Benavides (1552- $₫$ ?), compañero de Juan Cobo en su misionariado a las Filipinas, del que hablaremos en seguida. Benavides está considerado como el primer occidental que aprendió la lengua china, el primer sinólogo cuyo conocimiento de la lengua dejó plasmado en Vocabulario chino muy fácil (s.f.).

Pero quizá el más conocido sea el fraile agustino Martín de Rada (1533-1578), natural de Pamplona, autor del primer léxico sobre el chino, Vocabulario de la lengua china (s.f.). Esta obra, de concepción didáctica, sirvió de manual de aprendizaje para los posteriores misioneros. Entre ellos se encuentra el madrileño Diego de Pantoja (15711618), "el único que se integró verdaderamente en la sociedad china, siendo tratado por los intelectuales como "confuciano de Occidente"" (Zhang, 2013: 165). Este jesuita poseía un alto conocimiento de la lengua china y era capaz de escribir en caracteres, habilidad que dejó reflejada en varias obras como el 《七克》 (1614) (Tratado de los siete pecados y virtudes), que obtuvo una gran acogida y sucesivas reediciones hasta bien entrado el siglo XX.

A finales del XVII, otro sinólogo eminente, el sevillano Francisco Varo (1627-1687), se preocupó por la enseñanza y comprensión de la lengua china y compuso el Arte de la lengua mandarina (1682), considerada como la primera gramática de chino mandarín, aunque la descripción que hace parece ser de una variedad hablada de esta lengua por aquella época. El autor también escribió un Vocabulario de lengua mandarina (1692), una Gramática española mandarina (s.f.) y otro Vocabulario de la lengua mandarina con estilo y vocablos con que se habla sin elegancia (s.f.).

En las Filipinas, se tradujo la primera obra literaria del chino a una lengua occidental, el Beng Sim Po Cam / Espejo rico del claro corazón de Juan Cobo (1592). Aunque nuestro autor no podía comprender los caracteres chinos, parece ser que manejó el chino oral: el autor se valió de un informante sangley para transcribir y traducir al español lo que éste le narraba. Existe incluso un manuscrito de 1595 dedicado a Felipe II que se conserva todavía en la Biblioteca Nacional de España. Esta 
obra se puede considerar como una recopilación de textos filosóficos del Confucianismo, y también del budismo y del taoísmo. Esta obra fue muy popular en China, es de fácil lectura y posee un carácter moral hacia el perfeccionamiento personal por preceptos.

El español, como comentábamos, era la lengua ecuménica en Europa en el siglo XVII y la difusión de los materiales chinos se realizaba a través de ella. De la gran cantidad de textos del XVI y XVII conservados sobre China y sobre su lengua, la mayoría se encuentran en nuestro idioma.

Existen otros autores que merecen una mención como el ecijano Juan Bautista de Morales (1597-1664), que dejó numerosos tratados y cartas, así como un Diccionario chino español (s.f.); el palentino Antonio de Santa María Caballero (1602-1669) nos dejó sus Relaciones entre las distintas órdenes religiosas de China (1662) y Sobre algunos problemas importantes de la provincia china (1668), al igual que otras obras que recogían el pensamiento confuciano como Resumen del comienzo y el fin de todas las cosas (1664) y el muy conocido Confucianismo celestial (s.f.). Por último, vamos a citar a peñafielense Domingo Fernández Navarrete (1610-1689) con sus Controversias antiguas y modernas de la misión de la Gran China (s.f.) y sus Tratados históricos, políticos, éticos y religiosos del Imperio Chino (s.f.), obra esta que podemos considerar como la culminación de los estudios sinológicos españoles del siglo XVII. Hay que considerar todas estas obras en su justo valor, como obras únicas de difícil composición y transmisión debido a la dificultad que entraña la lengua china.

No obstante, paulatinamente, desde la segunda mitad del siglo XVII comienza a decrecer el poder de España como potencia hegemónica. Las raíces del contacto cultural habido entre los misioneros y China dejó una profunda huella en Europa y en los primeros representantes de la Ilustración temprana, como Leibniz, Voltaire y Montesquieu, intelectuales todos ellos que tuvieron noticia sobre el sistema político chino basado en los méritos a la persona para concurrir a los cargos públicos, al igual que la orientación hacia la Virtud promovida por el Confucianismo, frente a la búsqueda de la Verdad, objetivo del Cristianismo y de otras religiones. Especial éxito e influencia tuvo también el Arte de la guerra de Sunzi, traducido y reeditado continuamente. Todo ello conformó una visión sobre China en extremo positiva en la Europa ilustrada que se reflejará artísticamente en las conocidas en Francia como 
chinoiserie -chinerías en España-, el gusto por el arte chino: cerámicas, lacas, la seda, el té, la pintura, etc. Hay que resaltar que esta visión positiva de China acabará perdiéndose un siglo después cuando los contactos pasen a ser dominados por la británica East India Company.

\section{El predominio de la temática confuciana}

Otro capítulo aparte merece las obras traducidas a lenguas europeas, ante todo, traducciones sobre el confucianismo, ideología dominante en la época feudal china y que pasa a la modernidad como la esencia de la cultura china, adoptada por la doctrina política, la moral, etc. La urgencia de las misiones evangelizadoras y, más tarde, de las comerciales conllevaron el reto de conocer China en un breve lapso de tiempo y comprender su pensamiento. Se intentó siempre buscar paralelismos entre el pensamiento confuciano y el religioso cristiano, con el objetivo último de evangelizar China, como comentábamos.

Entre estas traducciones se encuentran obras como las siguientes:

- Michele Ruggleri 罗明坚 (1543-1607, Spinazzola, Italia), que tradujo Gran saber 《大学》, Mencio 《孟子》, publicados en Bibliotheca selecta qua agitur de ratione studiorum in historia, in disciplinis, in salute omnium procuranda (Roma, 1653).

- Alvaro Semedo 曾德昭 (1585-1658, portugués), que tradujo Imperio delle China 《大中国志》 (Madrid, 1642) y Libro de Cambio 《易经》.

- Ignacio a Costa y P. Prosper Intercetta 殷铎泽 (1625-1696, Italia), tradujeron Sapientia Sinica (que incluye Gran sabio 《大 学》〉, Jiangxi, 1662).

- P. Prosper Intercetta 殷铎泽 (1625-1696, Italia), tradujo Sinarum Scientia politico-moralis 《中庸》 (1667, Guangzhou).

- P. Prosper Intercetta, Christiani Herdtrich, Francisci Rougemont, Philippi Couplet, Patrum Societatis Jesu tradujeron Confucius Sinarum Philosophus, sive Scientia Sinensis 《中国哲学家孔 子》 (París, 1687).

- P. Franciscus Noël 卫方济 (1651-1729, Bélgica), que tradujo Sinensis Imperii Libri Classici Sex 《中国典籍六种》 (1711, Praga), (1784, París), (1786, París). 
- Claude de Visdelou 刘应 (1656-1737, Francia), que en sus viajes por Beijing, Shanxi y Guangdong recopiló 500 volúmenes chinos que acabó por traer a Europa; tradujo Libro de Ritos 《礼 记》 (s.f.).

- Joachim Bouvet 白晋 (1656, Francia), que en su viaje a Ningbo y a Beijing recopiló 49 libros chinos que trajo a Francia; escribió $I$ Ching 《易经大意》 《诗经研究》 (Estudio de Libro de odas, 1723).

La recepción de la literatura china en Europa se centró sólo en un principio en su filosofía. El siglo XVIII, sin embargo, trae consigo la difusión de la literatura china "pura", la de los géneros literarios. En Description géographique, historique, chronologique, politique, et physique de lémpeire de la Chine et de la Tartarie Chinoise (1735), el jesuita francés Jean-Baptiste Du Halde incluye en este volumen varias novelas cortas, cuentos y una decena de poemas del Libro de Odas, libro que posteriormente Goethe traducirá al alemán. Es precisamente a través de la lectura de la novela corta 《好述传》(The pleasing history), novela china de principios de la dinastía Qing, por la que Goethe anuncia que "La poesía es la posesión universal del ser humano", cita que inspiraría el concepto de Weltliteratur.

Otra obra china que tuvo gran repercusión fue L'Orphelin de la famille Zhao, obra teatral china de la dinastía Yuan (s. XIII). Esta pieza tendrá un enorme éxito durante el siglo XVIII y el XIX, traducida en una primera versión al francés por el misionero Joseph de Prénare en 1731. Del francés se vertió al inglés, alemán, ruso, italiano y otros idiomas. Hay que resaltar que fue en principio una traducción parcial, ya que Joseph de Prénare simplificó mucho la obra original, al igual que eliminó las canciones debido a la imposibilidad de traducirlas y que mantuvieran algo de su sonoridad. No obstante, una versión completa fue traducida por el sinólogo francés Stanislas Julien en 1834. Igualmente, aparecieron algunas adaptaciones en las décadas siguientes, como dos diferentes versiones en Inglaterra, la de William Hatchett (1741) y la de Arthur Murphy (1756); también hubo una versión en Italia que realizó Pietro Metastasio (1748). La adaptación de Goethe se publicó en 1748; la de Voltaire, en 1755. La difusión de esta obra teatral en Europa tiene su razón de ser en el valor de los pensamientos que encierra y que 
transmite, y no tanto, como ha resaltado la crítica, por su valor como obra literaria estética.

Al entrar el siglo XX, frente a una crisis de expresión artística, la literatura europea torna su mirada hacia las culturas orientales como horizontes alternativos para una renovación e innovación de sus propias letras. También le ocurrió a la literatura española, tal como advierte Andrés Herrera Feligreras (2007: 258):

A lo largo del diecinueve se inició un proceso por el cual España, como Estado, fue insertándose en el sistema de alianzas anglo-francés, y su intelligentsia, a falta de modelos propios, miraba más allá de Occidente a través de los ojos de orientalismo francés y británico, y por tanto con el filtro distorsionador del sentimiento de superioridad de lo occidental.

Son varios los factores que hay que considerar para entender la recepción de la Literatura china en España: las traducciones de obras literarias, los contactos directos e indirectos con la cultura oriental y la denominada mirada al poder y al prestigio. Según comenta Miner (2002: 187):

Cuando una nación o una cultura posee el poder según prestigio cultural, los escritores de otros países o de otras culturas están dispuestos a la recepción.

La recepción en el siglo XIX se convierte prácticamente en silenciosa acorde con la decadencia de China. La recepción, por lo tanto, cuando la hubo, fue sobre su pasado glorioso, sobre la sabiduría antigua y sobre su poesía, lo que se tradujo en una omisión o inconsciencia sobre su producción escrita de carácter novelístico. Aquellas obras que se tradujeron, incluso, fueron consideradas por la crítica china como de baja calidad, caso de las novelas cortas leídas por Goethe.

Otro de los problemas fundamentales fue la tendencia a traducir literalmente los poemas desde el chino. Tal como expresa Fenollosa (1977: 30): "Los sinólogos debieran recordar que el objeto de la traducción poética es la poesía y no las definiciones léxicas de los diccionarios". La configuración de la poesía china es tremendamente visual y musical, la traducción literal supone una doble pérdida de su valor original, lo que supone para el lector no chino una incomprensión sobre el valor real de la obra. 
Los préstamos literarios acabaron plasmándose en el Modernismo de fin de siglo, algunos de ellos, tópicos antiguos, pero que sirvieron de fuente de inspiración para reformar la estética literaria. Una muestra de ello puede considerarse la poesía de Valle-Inclán, admirador ferviente de Rubén Darío. En su poema "La tienda del herbolario" de La pipa del Kif (1919), China y sus tópicos se convierten en la máxima de la aspiración del decadentismo hacia la vida bohemia, espacio de huida y de ensoñaciones:
¡Adormideras! Feliz neblina, humo de opio que ama la China.
El opio evoca sueños azules, lacas, tortugas, leves chaúles. Ojos pintados, pies imposibles, lacias coletas, sables terribles. Verdes dragones, sombras chinescas, trágicas farsas funambulescas. Genuflexiones de Mandarines, sabias princesas en palanquines. Y nombres largos como poemas que evocan flores, astros y gemas.

Para Díaz Plaja (1979: 13):

Este "cuadro clínico" es producto, en gran parte, de "intoxicaciones" -bebidas, tabaco, opio, haschisch- de la vida de las grandes ciudades; de la fatiga que imprime la vida moderna y la conduce hasta los límites del agotamiento.

Las lecciones que se pueden sacar de los procesos históricos anteriores son alentadoras si se considera la perspectiva de la globalización, el cosmopolitismo y el comercio global: los desencuentros entre el Oriente Lejano y Occidente poseen una contraindicación clara para el devenir histórico actual; no hay otro camino sabio que el de aprendizaje mutuo, la adopción de ideas y la comprensión mutua de las distintas tradiciones. 


\section{Conclusiones}

La comunicación transcultural entre China y Occidente podría establecerse en tres simples edades o etapas:

- Del siglo XVI-XVIII: Europa muestra interés por la cultura china.

- Del siglo XIX-XX: A la inversa.

- Del siglo XX-Actualidad: Parece existir un interés recíproco.

A nuestro juicio, las interrelaciones existentes -la literatura sufre un camino tortuoso en este proceso- se pueden periodizar y denominar de la siguiente manera:

- Del siglo XVI-XVII: Periodo de la exploración: Conocimientos generales sobre China y su lengua, misiones evangelizadoras y traducciones de obras filosóficas.

- Siglo XVIII: Primer auge e influencia de la cultura china y del pensamiento filosófico chino en la Ilustración europea, época en que se producen traducciones literarias "puras" y recreaciones y adaptaciones de estas obras.

- Siglo XIX: Periodo de silencio: El interés se restringe entre los sinólogos debido a los problemas socioeconómicos producidos por el colonialismo. No obstante, se produce la fundación de algunas instituciones sinológicas.

- Siglo XX-XXI: Periodo de florecimiento: aumenta la cantidad de traducciones de obras orientales, se asienta la investigación de la sinología en las universidades europeas y también a la inversa, aumenta el número de publicaciones e investigaciones, se producen préstamos literarios, etc.

Dos sistemas culturales tan lejanos entre sí, de pensamiento y discurso social, hacen que la influencia literaria no sea un proceso de inmediatas asimilaciones, mucho más teniendo en cuenta el problema de la traductibilidad y los posibles malentendidos debido a la distancia existente entre los códigos lingüísticos y culturales. Uno de los factores determinantes es la actitud política y económica entre territorios en un momento histórico dado y que, pese al bache sufrido desde finales del XVIII, parece gozar en las últimas décadas de una vitalidad exce- 
lente. La característica intrínseca de la literatura es en el fondo la de proporcionar placer estético y cierta reflexión social; la globalización y la industrialización del fenómeno literario promueven la interacción cultural y el préstamo literario, tal como manifiesta Gálik (1994:18):

China and Europe are put on the two opposite ends of the Euroasian continent, but they are connected with innumerable treads in time and space in the most variegated realms, literary inclusive.

Lo interesante de este proceso es reconocer que cuenta con antecedentes explícitos en España, lo que nos sitúa en una posición privilegiada para afrontar el reto de la interculturalización.

\section{Bibliografía}

Barahona De Soto, Luis. 1981. Las lágrimas de Angélica. Madrid: Cátedra.

Cervantes Saavedra, Miguel de. 1978. El ingenioso hidalgo don Quijote de la Mancha. Barcelona: Andable.

Díaz Plaja, Guillermo. 1979. Modernismo frente a noventa y ocho. Madrid: Espasa-Calpe, S. A.

Fenollosa, Ernest F. \& Pound, Ezra. 1977. El carácter de la escritura china como medio poético. Ed. Visor: Madrid.

Herrera Feligreras, Andrés. 2007. La nueva sinología española. Huarte de San Juan. Geografía e Historia 14: 257-267.

Gálik, Marian. 1994. Chinese Literature and European Context. Bratislava: Institute of Asian and African Studies of the Slovak Academy of Sciences.

Lee, Thomas H. C. (ed.). 1991. China and Europe images and influences in sixteenth to eighteenth centuries. Hongkong: The Chinese University Press.

Lope De Vega, Félix. 2009. Angelica en el Catay. Alicante: Biblioteca Virtual Miguel de Cervantes. En: http://www.cervantesvirtual.com/nd/ ark:/59851/bmcvt262 [Consulta: 8 de octubre de 2018].

Miner, Earl. 2002. Estudios Comparados interculturales. En Angenot, Marc; Bessière, Jean; Fokkema, Douwe \& Kushner, Eva. Teoría literaria. México: Siglo XXI Editores, 183-205.

Ollé, Manel. 2000. La invención de China. Wiesbaden: Otto Harrassowitz.

Sanz, Carlos. 1958. Primitivas relaciones de España con Asia y Oceanía: los dos primeros libros impresos en Filipinas, más un tercero en discordia. Madrid: Librería General Victoriano Suárez. 
Valle-Inclán, Ramón del. 2017. Ligazón \& La pipa de kif. Madrid: Círculo de Bellas Artes.

Zhang, Kai. 2013. Historia de las relaciones sino-españoles. Beijing: China Intercontinental Press. 